\title{
Correlation of biofilm production with antibiotic susceptibility pattern of Pseudomonas aeruginosa from various clinical specimens
}

\author{
Swapna $\mathbf{M}^{1}$, Sumathi $\mathbf{G}^{2}$, Anitha $\mathbf{M}^{3}$ \\ ${ }^{1}$ Assistant Professor, Department of Microbiology, Vels Medical College and Hospital, Vels University, Manjakaranai, Thiruvallur \\ district, Tamil Nadu, India, ${ }^{2}$ Professor and Head, Department of Microbiology, Sri Muthukumaran Medical College Hospital and \\ Research Institute, Dr. M.G.R Medical University, Chikkarayapuram, Chennai, India, ${ }^{3}$ Lecturer, Department of Microbiology, Shri \\ Sathya Sai Medical College and Research Institute, Sri Balaji Vidyapeeth University, Thiruporur, Tamil Nadu, India
}

Background: Pseudomonas aeruginosa is one of the most prevalent nosocomial pathogens that cause a life-threatening infection. One of the important characteristics of $P$. aeruginosa is biofilm formation which leads to antibiotic resistance. Aims and Objectives: The aim of the study was to study the antibiotic resistance pattern of $P$. aeruginosa isolates and correlation with their biofilm-production. Materials and Methods: A total of $87 P$. aeruginosa isolates from different clinical specimens were processed and confirmed by conventional microbiological methods as per standard methodology. Antibiotic sensitivity testing was done for all isolates. Biofilm producing isolates were identified by the microtiter plate method (MTPM). Results: Of 87 P. aeruginosa isolates, majority were from pus $33(38 \%)$, followed by urine $26(30 \%)$, sputum $19(22 \%)$, body fluids $7(8 \%)$, and blood $2(2 \%)$. Biofilm producing isolates showed more resistance in comparison to non-biofilm producers. The observed difference between biofilm formation for multidrug resistant and susceptible isolates was found to be statistically significant. Conclusion: MTPM method was an effective test for detection of biofilm formation and was also able to verify biofilm production by $P$. aeruginosa. This indicated a higher propensity among the clinical isolates of $P$. aeruginosa to form biofilm and revealed a positive correlation between biofilm formation and antibiotic resistance. This indicates the need for testing of even susceptible isolates for virulence factors such as biofilm production.

Key words: Antibiogram; Biofilm; Microtiter plate method; Pseudomonas aeruginosa

\section{Access this article online}

\section{Website:}

http://nepjol.info/index.php/AJMS DOI: 10.3126/ajms.v13i1.39915

E-ISSN: 2091-0576

P-ISSN: 2467-9100

Copyright (c) 2022 Asian Journal of Medical Sciences

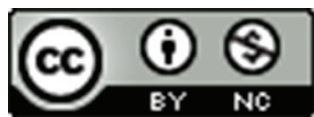

This work is licensed under a Creative Commons Attribution-NonCommercial 4.0 International License.

\section{INTRODUCTION}

Pseudomonas aeruginosa is ubiquitous Gram-negative bacilli, aerobic, and non-fermentative bacterium belonging to the family Psendomonadaceae, Psendomonas that is able to survive in a wide range of environments. ${ }^{1}$ Pseudomonas species, most especially the opportunistic pathogen $P$. aeruginosa, are known to exhibit large intrinsic resistance to multiple antibiotics across most classes including aminoglycoside, fluoroquinolones, and $\beta$-lactams (third and fourth generation cephalosporins, carbapenem, and monobactam).
Multidrug-resistant (MDR) P. aeruginosa strains have been implicated in urinary tract infections, bacteremia, respiratory tract infections, and wound infections. ${ }^{2}$ Other essential infections caused by the organism are pneumonia, endocarditis, endophthalmitis, meningitis, septicemia, and conjunctivitis. The frequency of $P$. aeruginosa was more in surgical and burn wound infections. ${ }^{3}$

P. aeruginosa is one of the most adaptive prevalent nosocomial pathogens. It has been implicated in serious and life-threatening infections. ${ }^{4}$ Infections caused by $P$. aeruginosa are associated with a higher death rate 
particularly in clinical settings. ${ }^{5}$ P. aeruginosa is an important etiological agent associated with healthcare-related infections and it has been shown to increase the rate of mortality and morbidity in patients. It can potentially become MDR due to its ability to acquire different antimicrobial resistance mechanisms. ${ }^{6}$

The pathogen shows the ability to produce biofilms, which are an important factor for virulence and bacterial resistance, and can have a strong impact on the health of the host. The dense polysaccharide matrix of the biofilm contributes to the persistence of infection, the ineffective action of antimicrobials, and the escape from the phagocytic actions of the cells of the immune system of the host, these effects result in chronic infections. ${ }^{7}$ Biofilms are micro-colonies composed of multiple microbial species formed during harsh conditions helping the survival of the microorganisms. ${ }^{8}$

This study was undertaken to investigate the antimicrobial resistance profile among various clinical isolates. In addition, in vitro biofilm-forming capabilities of $P$. aeruginosa isolated from clinical specimens were identified by microtiter plate method for biofilm production.

\section{Aims and objectives}

The aim of the study was to study the antibiotic resistance pattern of $P$. aeruginosa isolates and correlation with their biofilm-production.

\section{MATERIALS AND METHODS}

\section{Specimen collection}

This prospective study was carried out in department of microbiology, in a tertiary care hospital in Kancheepuram district. The samples were collected for a period of 6 months from January 2021 to June 2021. Informed consent was obtained from the patients before collecting the samples. A total of 311 clinical samples were collected from patients admitted in various wards of the hospital, among which 87 P. aeruginosa were isolated from pus, urine, sputum, body fluids, and blood samples. Seventy were MDR $P$. aeruginosa and 17 isolates were susceptible to various antibiotics. Ethical clearance was obtained from the institutional ethical committee.

\section{Bacterial identification}

All samples were cultured on multiple media (Nutrient agar, Blood agar, MacConkey agar, and Cetrimide agar). To identify the bacteria, pure colonies were processed for appropriate phenotypic characterization based on morphology, culture and further tested by conventional biochemical tests including catalase test, oxidase test, lactose fermentation test, hemolysin production test, and pigment production test and growth at $42^{\circ} \mathrm{C}$ leading to identification as P. aeruginosa.

\section{Antibiotic susceptibility testing}

Antibiotic sensitivity was tested by Kirby-Bauer disk diffusion method on Mueller-Hinton agar using antibiotic discs from Hi Media Laboratories (India) and the results were interpreted according to the criteria prescribed by Clinical and Laboratory Standards Institute. Antibiotics tested were: Amikacin $(30 \mu \mathrm{g})$, Cefuroxime $(30 \mu \mathrm{g})$, Ceftazidime $(30 \mu \mathrm{g})$, Cefipime $(30 \mu \mathrm{g})$, Gentamicin $(10 \mu \mathrm{g})$, Ciprofloxacin $(5 \mu \mathrm{g})$, Norfloxacin $(10 \mu \mathrm{g})$, Netilmycin $(30 \mu \mathrm{g})$, Piperacillin-Tazobactum $(100 / 10 \mu \mathrm{g})$, and Imipenem $(10 \mu \mathrm{g})$. P. aeruginosa ATCC 27853 was used as a control strain. ${ }^{9}$

\section{Detection of biofilm formation}

All bacterial isolates were tested by microtiter plate method (MTPM) for detection of biofilm formation.

\section{MTPMI (quantitative assay)}

P. aeruginosa biofilm was measured by MTPM to determine biofilm production. In this method, $P$. aeruginosa isolates were grown overnight at $37^{\circ} \mathrm{C}$ in Mueller-Hinton Broth containing $1 \%$ glucose. Then, microtiter plates were inoculated with $125 \mu \mathrm{l}$ bacterial suspension and adjusted to $0.5 \mathrm{McF}$ arland. Microtiter plates were incubated for $24 \mathrm{~h}$ at $37^{\circ} \mathrm{C}$. Biofilms formed on the walls of microtiter plate were stained with $150 \mu \mathrm{l}$ of $0.1 \%$ crystal violet for $10 \mathrm{~min}$. Then, plates were washed twice with phosphatebuffered saline ( $\mathrm{pH} 7.2)$ to discharge crystal violet stain. After air drying, microplate was re-solubilized by $150 \mu \mathrm{l}$ of $95 \%$ ethanol. Then, plate was measured at $570 \mathrm{~nm}$ by a microtiter plate reader. ${ }^{10}$

Statistical analysis between MDR and susceptible isolates for biofilm formation

Chi-square test was applied to find out the association between MDR and susceptible isolates for biofilm production. The observed difference between biofilm formation and MDR and susceptible isolates were found to be statistically significant. $\mathrm{P}<0.05$ was considered as statistically significant.

\section{RESULTS}

During the 6 months period of study from January 2021 to June 2021, 87 clinical isolates of P. aeruginosa were collected from various clinical samples. The phenotypic identification of the $P$. aeruginosa isolates was performed by bacteriological methods (Grams staining, colony morphology, and biochemical tests) using standard methodology. Of 87 sample, $49(56 \%)$ were from males and 38 (44\%) were from females (Table 1, Figures 1 and 2). 
The maximum number of isolates was obtained from pus $33(38 \%)$, followed by urine $26(30 \%)$, sputum $19(22 \%)$, body fluids $7(8 \%)$, and blood $2(2 \%)$. In the present study, $P$. aeruginosa showed resistance against most of the commonly used antibiotics (Table 2). Out of 87 isolates, $70(80 \%)$ of $P$. aeruginosa isolates were identified as MDR and $17(20 \%)$ of isolates were susceptible to most commonly used antibiotics (Table 3).

All 87 isolates were tested for biofilm production by MTPM. Among the MDR isolates, 68 (97\%) were biofilm producers and $2(3 \%)$ were biofilm non-producers. MDR

\begin{tabular}{lcc}
$\begin{array}{l}\text { Table 1: Gender-wise distribution of } \\
\text { Pseudomonas aeruginosa }\end{array}$ \\
\hline Clinical samples & No. of Isolates & Percentage \\
\hline Pus & 33 & 38 \\
Urine & 26 & 30 \\
Sputum & 19 & 22 \\
Body fluids & 7 & 8 \\
Blood & 2 & 2 \\
\hline
\end{tabular}

\begin{tabular}{|c|c|c|}
\hline \multicolumn{3}{|c|}{$\begin{array}{l}\text { Table 2: Distribution of Pseudomonas } \\
\text { aeruginosa from various clinical } \\
\text { specimens }(n=87)\end{array}$} \\
\hline Gender & No of isolates & Percentage \\
\hline & 49 & 56 \\
\hline Female & 38 & 44 \\
\hline
\end{tabular}

\begin{tabular}{|c|c|c|}
\hline Susceptibility pattern & No. of isolates & Percentage \\
\hline Sensitive & 17 & 20 \\
\hline MDR & 70 & 80 \\
\hline Total & 87 & 100 \\
\hline
\end{tabular}

\begin{tabular}{|c|c|c|c|}
\hline \multicolumn{4}{|c|}{ MTPM } \\
\hline Biofilm producer & Percentage & $\begin{array}{l}\text { Non-biofilm } \\
\text { producer }\end{array}$ & Percentage \\
\hline 68 & 97 & 2 & 3 \\
\hline
\end{tabular}

\begin{tabular}{|c|c|c|c|}
\hline \multicolumn{4}{|c|}{ MTPM } \\
\hline $\begin{array}{l}\text { Biofilm } \\
\text { producer }\end{array}$ & Percentage & $\begin{array}{l}\text { Non-biofilm } \\
\text { producer }\end{array}$ & Percentage \\
\hline 4 & 24 & 13 & 76 \\
\hline
\end{tabular}

isolates showed maximum positivity for biofilm formation in MTPM. There was a significant relationship between biofilm production and MDR. In addition, biofilm formation was verified for 17 antibiotic susceptible isolates and the results of MTPM showed 4 (24\%) were biofilm producers and $13(76 \%)$ were biofilm non-producers. There was a significant difference between biofilm production and susceptible isolates. Biofilm producing isolates showed more resistance in comparison to non-biofilm producers (Tables 4 and 5, Figure 3).

This study shows the need for testing of susceptible isolates for presence of virulence factors such as biofilm production which will help in choosing appropriate antibiotics to treat patients with $P$. aeruginosa infections.

\section{DISCUSSION}

The World Health Organization (WHO) declared P. aeruginosa as a priority among the present pathogens urgently in need

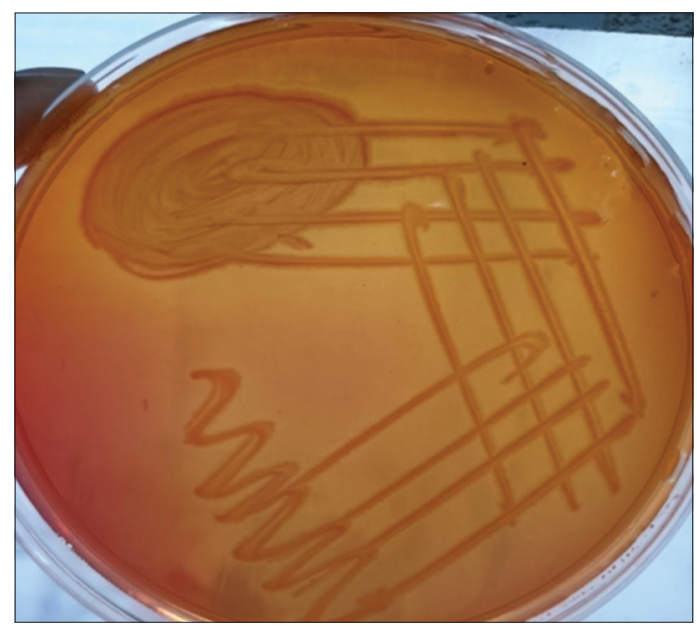

Figure 1: Non-lactose fermenting on MacConkey agar

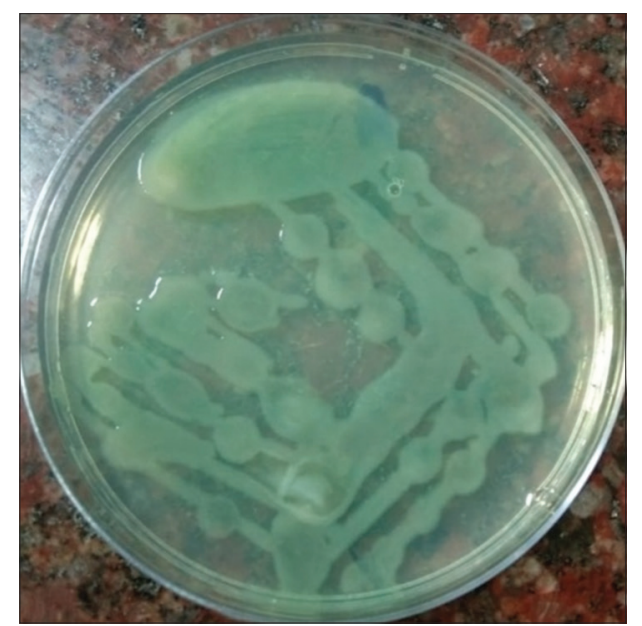

Figure 2: Pigment production on nutrient agar

Asian Journal of Medical Sciences | Jan 2022 | Vol 13 | Issue 1 


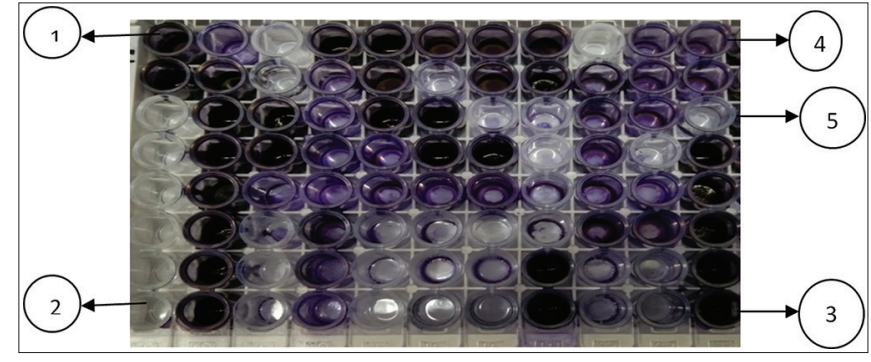

Figure 3: Screening of biofilm producers by microtiter plate method. (1) Positive control, (2) negative control, (3) strong biofilm producer, (4) moderate biofilm producer, and (5) weak biofilm producer

of new effective antibiotics (the WHO, 2020). ${ }^{11}$ In this study, of a total of 87 isolates, the antibiotic susceptibility pattern of $P$. aeruginosa revealed that isolates were predominantly resistant showing MDR pattern 70 (80\%). Only $17(20 \%)$ were sensitive to commonly used antibiotics. These results are similar with the study conducted by Ijaz et al. ${ }^{12}$ who showed resistance pattern 119 (58.6\%).

Gender distribution shows male $(58 \%)$ predominance over female $(42 \%)$ in this study. Similar observation was made in other studies that reported a slight male preponderance, ${ }^{13,14}$ whereas Anil and Shahid have reported slight predominance of female patients $80(55.17 \%)$ over males $65(44.83 \%))^{15}$

In the present study, 87 P. aeruginosa were isolated from various clinical samples. About $33(38 \%)$ isolates were from pus samples, followed by urine $26(30 \%)$, sputum $19(22 \%), 7$ (8\%) from body fluids, and $2(2 \%)$ blood samples. Similarly in another study by Golia et al., majority of isolates were from pus samples $67(55.83 \%) .{ }^{13}$ In contrast, Rodrigues et al. reported that maximum isolation rate of $P$. aeruginosa from blood (33.3\%), tracheal secretion $(23.8 \%)$, and urine $(23.8 \%)$ was the most prevalent sources of $P$. aeruginosa. ${ }^{16}$

There was a high prevalence of biofilm production in these isolates. Furthermore, Haji reported that, out of 96, 84 (87.5) isolates were biofilm producers by MTPM method. ${ }^{17}$ MTPM method is also reported as gold standard by other researchers Karthic and Gopinath, ${ }^{5} \mathrm{Haji} .{ }^{17}$ Hence, MTPM method was considered as standard method for additional interpretation of results. The major problem attributed with infections formed by biofilm producer bacteria is abundance of resistance to various antibiotics. ${ }^{5}$

It is evident that there was a high frequency of resistance against all the commonly used antimicrobial agents. This observation is supported by various other researchers. ${ }^{5,18}$

The ability all isolates of $P$. aeruginosa to produce biofilm was detected using standard microtiter plates. All $P$. aeruginosa isolates had the ability of biofilm production.
The association between biofilm formation and antibiotic resistance revealed that biofilm production was statistically significant among MDR and susceptible P. aeruginosa isolates this agreement with Ismail and Altaai. ${ }^{19}$

In the present study, among a total of 87 isolates of P. aeruginosa, 68 (97\%) were biofilm-producers and this finding is comparable with a study done by Neopane et al., ${ }^{20}$ who showed $(83.33 \%)$, but in contrast with others who showed lower rate of biofilm production $(33 \%)^{21}$ and $22(26.3 \%) .^{22}$

For confirmation of virulence factors produced by both MDR and susceptible isolates of $P$. aeruginosa, molecular techniques will be helpful. Hence, in future, such studies can be done using molecular techniques, which will help in proper antibiotic treatment.

\section{Limitations of the study}

The study period only for six months, so the number of samples were less. Further study needs to be conducted in the same line to establish the facts.

\section{CONCLUSION}

Most of the $P$. aeruginosa isolates showed resistance to a wide range of antibiotics. This study shows a high incidence of biofilm productions among $P$. aeruginosa isolates. MTPM method was considered as effective test for detection of biofilm formation by $P$. aeruginosa. Importantly, $P$. aeruginosa isolates were observed to be resistant to most commonly used antimicrobials. This indicated a higher propensity among the clinical isolates of $P$. aeruginosa to form biofilm and there was a positive correlation between biofilm formation and antibiotic resistance. Even susceptible strains were shown to produce biofilm. This study shows the need for testing of even susceptible isolates for the presence of virulence factors which will help in choosing appropriate antibiotics to treat patients with $P$. aeruginosa infections. Based on findings of this study, it is recommended that MTPM method can be used as a screening method. Furthermore, this study indicates need for further molecular support for virulence testing of all isolates of $P$. aeruginosa.

\section{ACKNOWLEDGMENT}

We extend our heartfelt gratitude to the hospital and management for their support to carry out this project.

\section{REFERENCES}

1. Silby MW, Winstanley C, Godfrey SA, Levy SB and Jackson RW. Pseudomonas genomes: Diverse and adaptable. FEMS Microbiol Rev. 2011;35(4):652-680. 
https://doi.org/10.1111/j.1574-6976.2011.00269.x

2. Curran CS, Bolig $T$ and Torabi-Parizi P. Mechanisms and targeted therapies for Pseudomonas aeruginosa lung infection. Am J Respir Crit Care Med. 2018;197(6):708-727.

https://doi.org/10.1164/rccm.201705-1043so

3. Meskini M and Esmaeili D. The study of formulated Zoush ointment against wound infection and gene expression of virulence factors Pseudomonas aeruginosa. BMC Complement Altern Med. 2018;18(1):185.

https://doi.org/10.1186/s12906-018-2251-4

4. Fu B, Wu Q, Dang M, Bai D, Guo Q, Shen L, et al. Inhibition of Pseudomonas aeruginosa biofilm formation by traditional Chinese medicinal herb herba patriniae. Biomed Res Int. 2017;2017:9584703.

https://doi.org/10.1155/2017/9584703

5. Karthic A and Gopinath P. Detection of biofilm among clinical isolates of Pseudomonas aeruginosa by tissue culture plate (TCP) method. J Chem Pharm Sci. 2016;9(4):3236-3238.

6. Kırmusaoglu S, Yurdugül S, Metin A and Vehid S. The effect of urinary catheters on microbial biofilms and catheter associated urinary tract infections. Urol J. 2017;14(2):3028-3034.

7. Asati $S$ and Chaudhary U. Prevalence of biofilm producing aerobic bacterial isolates in burn wound infections at a tertiary care hospital in northern India. Ann Burns Fire Disasters. 2017;30(1):39-42.

8. Frösler J, Panitz C, Wingender J, Flemming HC and Rettberg P. Survival of Deinococcus geothermalis in biofilms under desiccation and simulated space and Martian conditions. Astrobiology. 2017;17(5):431-447.

https://doi.org/10.1089/ast.2015.1431

9. CLSI. Performance Standards for Antimicrobial Susceptibility Testing. CLSI Supplement M100S. 29 ${ }^{\text {th }}$ ed., Vol. 36. Wayne, PA: Clinical and Laboratory Standards Institute; 2019. p. 40-256. Available from: https://www.clsi.org/media/26663/m100ed29. [Last accessed on 2021 Jan 19]. https://doi.org/10.1201/9781420014495-6

10. Jaffar $\mathrm{N}$, Miyazaki $\mathrm{T}$ and Maeda $\mathrm{T}$. Biofilm formation of periodontal pathogens on hydroxyapatite surfaces: Implications for periodontium damage. J Biomed Mater Res A. 2016;104(11):2873-2880.

https://doi.org/10.1002/jbm.a.35827

11. Beyer $P$ and Paulin S. Priority pathogens and the antibiotic pipeline: An update. Bull World Health Organ. 2020;98(3):151. https://doi.org/10.2471/blt.20.251751

12. ljaz $M$, Siddique $A B$, Rasool $M H$ and Shafique $M$. Frequency of multi drug resistant Pseudomonas aeruginosa in different wound types of hospitalized patients. Pak J Pharm Sci. 2019;32 Suppl 2:865-870.

13. Golia S, Suhani S, Manasa S and Jyothi S. Isolation of pseudomonas aeruginosa from various clinical isolates and it antimicrobial resistance pattern in a tertiary care hospital. Int $\mathrm{J}$ Curr Microbiol Appl Sci. 2016;5(3):247-253. https://doi.org/10.20546/ijcmas.2016.503.030

14. Kamali E, Jamali A, Ardebili A Ezadi F and Mohebbi A. Evaluation of antimicrobial resistance, biofilm forming potential, and the presence of biofilm-related genes among clinical isolates of Pseudomonas aeruginosa. BMC Res Notes. 2020;13:27. https://doi.org/10.1186/s13104-020-4890-z

15. Anil C and Shahid RM. Antimicrobial susceptibility patterns of Pseudomonas aeruginosa clinical isolates at a tertiary care hospital in Kathmandu, Nepal. Asian $\mathrm{J}$ Pharm Clin Res. 2013;6(3):235-238.

16. Rodrigues RL, da Costa Lima JL, de Sena KX and Maciel MA. Phenotypic and genotypic analysis of biofilm production by Pseudomonas aeruginosa isolates from infection and colonization samples. Rev Soc Bras Med Trop. 2020;53:e20200399. https://doi.org/10.1590/0037-8682-0399-2020

17. Haji SH. Detection of biofilm formation in Pseudomonas aeruginosa Isolates from clinical specimens. Official Sci J Salahaddin Univ Erbil ZJPAS. 2018;30(4):83-89.

https://doi.org/10.21271/zjpas.30.4.9

18. Bakir SH and Ali FA. Comparison of different methods for detection of biofilm production in multi-drug resistance bacteria causing pharyngotonsillitis. Int $\mathrm{J}$ Res Pharm Biosci. 2016;3(2):13-22.

19. Ismail ST and Altaai MI. Study ndvB gene expression in Pseudomonas aeruginosa producing biofilm. Med Legal Update. 2021;21(1):961-965.

https://doi.org/10.37506/mlu.v21i1.2440

20. Neopane P, Nepal HP, Gautam R, Paudel R, Ansari S, Shrestha S, et al. Is there correlation of biofilm formation with multidrug resistance and ESBL production in pseudomonas aeruginosa. Eur J Biomed Pharm Sci. 2017;4(1):366-372. Available from: https://www.ejbps.com. [Last accessed on 2021 Jan 19].

21. Shrestha R, Nayak N, Bhatta DR, Hamal D, Subramanya SH and Gokhale S. Drug resistance and biofilm production among Pseudomonas aeruginosa clinical isolates in a tertiary care hospital of Nepal. Nepal Med Coll J. 2019;21(2):110-116. https://doi.org/10.3126/nmcj.v21i2.25109

22. Kulkarni DM, Nilekar SL and Vidhya T. Association of biofilm production in ESBL and MBL producing clinical isolates of Pseudomonas aeruginosa. Trop $\mathrm{J}$ Pathol Microbiol. 2020;6(2):174-180.

\footnotetext{
Authors Contribution:

SM- Conceptualization, study design, literature review, prepared first draft of manuscript, and interpreted the results; SG- revision of the manuscript, manuscript drafting; AM- Data collection, data analysis, and tabulation of results

Work attributed to:

Sri Muthukumaran Medical College Hospital and Research Institute, Dr. M.G.R Medical University, Chikkarayapuram, Tamil Nadu, India

Orcid ID:

Dr. Swapna M - (i) https://orcid.org/0000-0003-2628-4905

Dr. Sumathi G - (1) https://orcid.org/0000-0002-1731-4394

Dr. Anitha M - (1) https://orcid.org/0000-0003-1440-7218

Source of Funding: None, Conflicts of Interest: None.
} 